\title{
Elastase Inhibitory Activity of Airway $\alpha 1$-Antitrypsin Is Protected by Treatment With a Catalytic Antioxidant in a Baboon Model of Severe Bronchopulmonary Dysplasia
}

\author{
ÇAGATAY KARAASLAN, HIROSHI HIRAKAWA, RYUJI YASUMATSU, LING-YI L. CHANG, RICHARD A. PIERCE, \\ JAMES D. CRAPO, AND SULE CATALTEPE
}

\begin{abstract}
Division of Newborn Medicine [C.K., H.H., R.Y.], Brigham and Women's Hospital and Harvard Medical School, Boston, Massachusetts 02115; Department of Medicine [L.-Y.L.C., J.D.C.], National Jewish Medical and Research Center, Denver, Colorado 80206; Department of Internal Medicine [R.A.P.], Washington University School of Medicine, St. Louis, Missouri 63110
\end{abstract}

\begin{abstract}
Recent studies in animal models of bronchopulmonary dysplasia (BPD) suggest that antioxidant treatments may be beneficial for the disease. However, the mechanisms by which these drugs improve the course of BPD are not completely known. Alpha1antitrypsin $(\alpha 1-\mathrm{AT})$ is one of the major serine protease inhibitors in human plasma that has antielastase and antiapoptotic activities. Both activities of $\alpha 1$-AT are dependent on its reactive site loop (RSL), which is highly susceptible to oxidative inactivation. In this study, we investigated the elastase inhibitory activity of $\alpha 1$-AT in two different baboon models of BPD, the "new BPD" and the "severe BPD" models, and determined the effect of treatment with a catalytic antioxidant, $\mathrm{Mn}(\mathrm{III})$ meso-tetrakis( $N$-ethylpyridinium-2-yl)porphyrin (MnTE-2-PyP), on the elastase inhibitory activity of $\alpha 1$-AT in the severe BPD model. Our results demonstrate the presence of sufficient elastase inhibitory activity of the airway $\alpha 1$-AT in the new but not in the severe BPD model. Treatment of severe BPD group baboons with the catalytic antioxidant MnTE-2-PyP resulted in augmentation of the elastase inhibitory activity of $\alpha 1$-AT. These findings suggest that prevention of the oxidative inactivation of $\alpha 1$-AT may be one of the mechanisms by which antioxidant therapy improves the pulmonary outcomes in animal models of severe BPD. (Pediatr Res 70: 363-367, 2011)
\end{abstract}

$\mathrm{B}$ ronchopulmonary dysplasia (BPD) remains as the most common complication of very preterm birth (reviewed in Refs. 1-5). Infants with BPD not only suffer from long-term pulmonary dysfunction but are also at higher risk of having growth restriction and adverse neurodevelopmental outcomes compared with age-matched infants $(6-11)$. The pathogenesis of BPD is multifactorial and complex. Barotrauma, volutrauma, oxygen toxicity, antenatal and postnatal inflammation, and patent ductus arteriosus have been implicated to play a role in the development of BPD (reviewed in Refs. 1, 5, and 12). An enhanced inflammatory reaction with persistent influx of neutrophils is observed in the airways of preterm infants, who subsequently develop BPD $(13,14)$. This inflammation is

Received February 9, 2011; accepted April 22, 2011.

Correspondence: Sule Cataltepe, M.D., Department of Newborn Medicine, Brigham and Women's Hospital, Thorn 1019, 75 Francis Street, Boston, MA 02115; e-mail: scataltepe@partners.org

Supported by NIH HL075904 [to S.C.] and NIH HL63397 [to J.D.C.].

C.K. is currently at the Department of Biology, Hacettepe University, Ankara, Turkey. H.H. is currently at the Department of Obstetrics and Gynecology, Chiba Aiyukai Memorial Hospital, Nagareyama City, Japan. R.Y. is currently at the Department of Otorhinolaryngology, Kyushu University, Fukuoka, Japan. associated with an abundance of reactive oxygen species and proteases that may not be sufficiently regulated by antioxidants and antiproteases, respectively, of the preterm lung (15-17).

Several studies in animal models of BPD have demonstrated structural and functional improvements with antioxidant treatments. Transgenic newborn mice that overexpress human extracellular superoxide dismutase (SOD) demonstrated reduced inflammation and improved epithelial cell proliferation and preservation of alveolar surface and volume density when exposed to hyperoxia $(18,19)$. In hyperoxiaexposed baboons, i.v. treatment with a catalytic antioxidant, MnTE-2-PyP [Mn(III) meso-tetrakis( $N$-ethylpyridinium-2yl)porphyrin], resulted in improved alveolar surface area and decreased parenchymal mast cells, eosinophils, and neuroendocrine cells and urine bombesin-like-peptide levels (20). In a multicenter trial, treatment of premature infants with intratracheal recombinant human CuZn superoxide dismutase (rCuZnSOD) failed to decrease the incidence of death or BPD but resulted in a significant decrease in the number of patients who required asthma medications, had wheezing episodes, required emergency room visits, or required rehospitalizations at 1 y corrected GA compared with the controls (21). Thus, although this study indicates that treatment with r-CuZnSOD may reduce lung injury, it is not clear why it did not have an effect on BPD incidence. Furthermore, the mechanisms by which antioxidant agents decrease inflammation and improve alveolarization in animal models are not completely understood.

Alpha1-antitrypsin $(\alpha 1-\mathrm{AT})$ is one of the major serine protease inhibitors (serpin) in human plasma and has been a molecule of interest in BPD as one of the major inhibitors of neutrophil elastase (NE). In a study by Stiskal et al. (22), i.v. administration of $\alpha 1$-AT to premature infants with RDS decreased the incidence of pulmonary hemorrhage without having an effect on the incidence of BPD. In addition to its antielastase activity, recent studies have also identified a novel role for $\alpha 1-\mathrm{AT}$ in apoptosis as an inhibitor of caspase-3

Abbreviations: $\alpha \mathbf{1 - A T}$, alpha1-antitrypsin; BALF, bronchoalveolar lavage fluid; BPD, bronchopulmonary dysplasia; GC, gestational control; MnTE-2PyP, Mn(III) meso-tetrakis( $N$-ethylpyridinium-2-yl)porphyrin; NE, neutrophil elastase; PRN, pro re nata; RSL, reactive site loop; RT, room temperature 
(23-25). Similar to its antielastase activity, the antiapoptotic activity of $\alpha 1-\mathrm{AT}$ is dependent on its reactive site loop (RSL), which is highly susceptible to oxidative inactivation (24). In this study, we investigated the elastase inhibitory activity of airway $\alpha 1-\mathrm{AT}$ in two different baboon models of BPD and determined the effect of the catalytic antioxidant, MnTE-2-PyP, on the elastase inhibitory activity of $\alpha 1$-AT recovered from the airways of baboons with hyperoxia-induced severe BPD.

\section{METHODS}

Animal model. Frozen baboon lung tissue and necropsy bronchoalveolar lavage fluid (BALF) samples were provided by the Southwest Foundation for Biomedical Research (San Antonio, TX). All animal procedures were reviewed and approved by the animal care committees of the Southwest Foundation for Biomedical Research and the University of Texas Health Science Center in San Antonio. In the new BPD model, baboons that were delivered by hysterotomy at $125 \mathrm{~d}$ were intubated, treated with exogenous surfactant (Survanta; donated by Ross Laboratories, Columbus, OH), and maintained on pressure-limited, time-cycled infant ventilators (donated by InfantStar; Infrasonics, San Diego, CA) for $2 \mathrm{~d}, 6 \mathrm{~d}$, or $14 \mathrm{~d}$ (new BPD group). The ventilator settings were adjusted to maintain the arterial carbon dioxide tension $\left(\mathrm{PaCO}_{2}\right)$ between 45 and $55 \mathrm{~mm} \mathrm{Hg}$, and oxygen was provided on a pro re nata $(\mathrm{PRN})$ basis to maintain the arterial oxygen tension $\left(\mathrm{PaO}_{2}\right)$ between 55 and $70 \mathrm{~mm} \mathrm{Hg}$. Animals that were killed at $14 \mathrm{~d}$ had pathologic and biochemical findings that were characteristic of the new BPD seen in human infants as described previously (26). Baboons that were delivered at $125 \mathrm{~d}$ or $140 \mathrm{~d}$ and killed immediately served as the gestational controls (125-d GC or 140-d GC groups). A third control group consisted of baboons that were born via natural delivery at full-term gestation $(\sim 185 \mathrm{~d})$ and killed $2-3 \mathrm{~d}$ later (full-term group). In the "severe BPD" model, baboons were delivered at $140 \mathrm{~d}$ gestation and were ventilated for a total of $10 \mathrm{~d}$ with $100 \% \mathrm{O}_{2}(27)$. The catalytic antioxidant MnTE-2-PyP was administered to seven baboons that were exposed to $100 \% \mathrm{O}_{2}$ continuously. As MnTE-2-PyP has a half-life of 0.5 to $1 \mathrm{~h}$ in mice, it was administered by continuous i.v. infusion at a dose of 0.5 $\mathrm{mg} / \mathrm{kg} / \mathrm{d}$ using an infusion rate of $0.1 \mathrm{~mL} / \mathrm{h}$ as previously described (20). A preliminary 2-wk toxicity study in mice using continuous infusion of the drug demonstrated no toxic effects at $5 \mathrm{mg} / \mathrm{kg} / \mathrm{d}$. Therefore, a 10 -fold lower dose was chosen for this initial study in immature baboons.

Isolation of total RNA and reverse transcription. Total RNA was isolated from fresh-frozen baboon lung (right middle lobe) or liver tissues using Trizol reagent (Invitrogen, Carlsbad, CA) and was treated with DNAse I (Invitrogen) following the manufacturer's instructions. First-strand cDNA was synthesized from $0.5 \mu \mathrm{g}$ of RNA using the Superscript First-Strand Synthesis System (Invitrogen) with $0.5 \mu \mathrm{g}$ oligo-dT. The reaction mixture was incubated at $42^{\circ} \mathrm{C}$ for $50 \mathrm{~min}$ followed by incubation at $72^{\circ} \mathrm{C}$ for $15 \mathrm{~min}$. cDNA was stored at $-20^{\circ} \mathrm{C}$ until use.

Real-time PCR. Real-time PCR analysis was performed using the Mx4000 Multiplex Quantitative PCR System (Stratagene Inc, LA Jolla, CA) and the brilliant SYBR Green QPCR Master Mix (Stratagene Inc). The sequences of PCR primers were designed using the IDT DNA web site (http:// www.idtdna.com) and were as follows: alpha1-antitrypsin forward primer, 5'-AGGAGCTTGACAGAGACACAGT-3', reverse primer, 5'-TCGGTGTCCTTGACTTCAAAGG-3'; cyclophilin A forward primer, 5'TTCATCTGCACTGCCAAGACTG-3', reverse primer, 5'-GGC CTC CAC AAT ATT CAT GCC T- $3^{\prime}$. For PCR analysis, $2 \mu \mathrm{L}$ cDNA was diluted 1:10, and the reactions were performed in $20 \mu \mathrm{L}$ of reaction volume with the following conditions: initial denaturation at $95^{\circ} \mathrm{C}$ for $10 \mathrm{~min}$, followed by 35 cycles of denaturation at $95^{\circ} \mathrm{C}$ for $30 \mathrm{~s}$, annealing at $62^{\circ} \mathrm{C}$ for $60 \mathrm{~s}$, and extension at $72^{\circ} \mathrm{C}$ for $30 \mathrm{~s}$. All reactions were performed in triplicates and repeated at least three times. Cyclophilin A was used as an internal reference to normalize the target transcripts using the $2^{-\Delta \Delta \mathrm{CT}}$ method $(28,29)$.

Baboon BALF. BALF samples were obtained at the time of necropsy. Sterile $0.9 \%$ saline was instilled into the left lower lobe until the lobe was completely filled and drawn back a total of five times. BALF samples were centrifuged and supernatants were stored at $-80^{\circ} \mathrm{C}$ until use. Total protein concentration was determined by Bradford's Assay (Bio-Rad, Hercules, CA). The concentration of each sample was adjusted to $0.8 \mu \mathrm{g} / \mu \mathrm{L}$ either by adding PBS or by concentrating the sample using a Vivaspin Concentrator (ISC Bio Express, Kaysville, UT) according to the manufacturer's protocol.

Determination of the elastase inhibitory activity of $\alpha 1-A T$ in $B A L F$ by formation of NE/ $\mathbf{I}-\mathbf{A T}$ complexes. BALFs $(10 \mu \mathrm{L}$, total protein concentration, $0.8 \mu \mathrm{g} / \mu \mathrm{L}$ ) were incubated with two different concentrations of purified $\mathrm{NE}(2 \mu \mathrm{L}$ of $16 \mathrm{ng} / \mu \mathrm{L}$ or $4 \mathrm{ng} / \mu \mathrm{L}$; Athens Research, Athens, GA) or the same

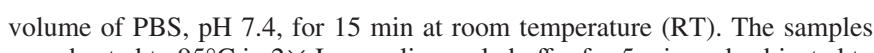
were heated to $95^{\circ} \mathrm{C}$ in $2 \times$ Laemmli sample buffer for $5 \mathrm{~min}$ and subjected to immunoblotting as previously described (16). Briefly, the proteins were separated by SDS-PAGE and transferred onto a nitrocellulose membrane. The membrane was blocked in a buffer containing PBS, $\mathrm{pH} 7.4,0.1 \%$ Tween-20, and $5 \%$ dried milk for $1 \mathrm{~h}$ and incubated with a rabbit anti-human $\alpha 1-\mathrm{AT}$ polyclonal antibody at a dilution of 1 in 8000 (Sigma Chemical Co., St. Louis, MO) for $1 \mathrm{~h}$ at RT. Subsequently, the membrane was rinsed in wash buffer and incubated for $1 \mathrm{~h}$ with horseradish peroxidase-conjugated anti-rabbit IgG (Jackson Immunoresearch Laboratories, Westgrove, PA). After three rinses in wash buffer, the protein bands were visualized by chemiluminescence (ECL Western Blotting Analysis System; Amersham Biosciences, Piscataway, NJ).

Statistical analysis. Mann-Whitney $U$ test and Fisher's exact test were used for analysis of nonparametric and categorical data, respectively. Noncategorical data are presented as mean $\pm \mathrm{SEM}$, and $p<0.05$ is considered to be significant.

\section{RESULTS}

Effect of GA and BPD on airway $\alpha 1$-antitrypsin. We first determined the relative antigenic levels of $\alpha 1$-AT in necropsy BALF obtained from premature and full-term control baboons and baboons with BPD by immunoblotting. Control premature baboons were delivered and killed at $125 \mathrm{~d}$ or $140 \mathrm{~d}$. Bronchopulmonary dysplasia groups included two different models. In the first model, animals were delivered at $125 \mathrm{~d}$ and treated with one dose of surfactant, mechanical ventilation and as needed (PRN) $\mathrm{O}_{2}$ for $14 \mathrm{~d}$. This model induces lung injury that recapitulates the interrupted alveolarization and vascularization observed in human infants with "new BPD." In the second model, baboons were delivered at 140-d gestation and exposed to $100 \% \mathrm{O}_{2}$ for $10 \mathrm{~d}$. This model results in lung injury that is consistent with hyperoxia-induced severe BPD. Seven baboons from this group were treated with the catalytic antioxidant MnTE-2-PyP as a continuous infusion for $10 \mathrm{~d}$.

Alpha1-antitrypsin was detected primarily as a $52 \mathrm{kD}$ band in all control BALF samples (Fig. 1, black arrow). All samples also contained lower molecular mass bands, likely due to proteolytic cleavage of $\alpha 1$-AT (Fig. 1, white arrow). In both BPD groups, there were increased levels of $\alpha 1$-AT compared with the control samples. In addition, BPD samples demonstrated more cleavage products compared with the control samples. There were no obvious differences in quantity or quality of $\alpha 1$-AT bands between the $125-\mathrm{d} / \mathrm{PRN} \mathrm{O}_{2}$ and $140-\mathrm{d} / 100 \% \mathrm{O}_{2}$ BPD models.

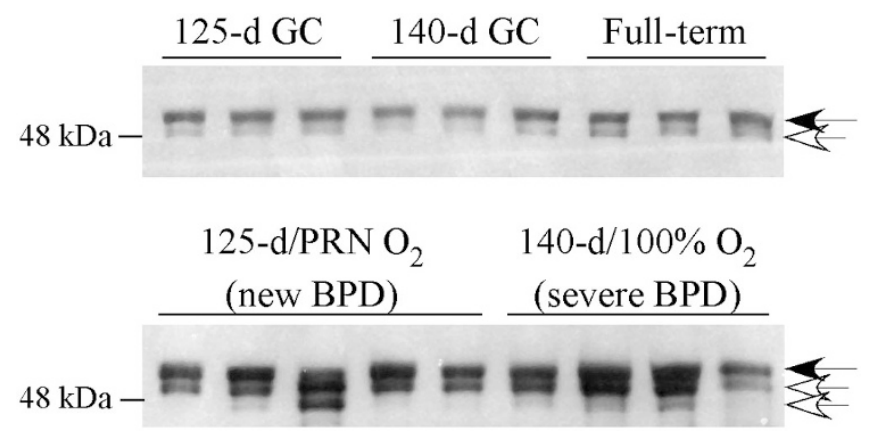

Figure 1. Alpha1-antitrypsin in baboon BALF samples. BALF samples were collected at the time of necropsy from $125 \mathrm{~d}, 140 \mathrm{~d}$, and full-term gestation baboons and baboons with new (125-d/14 d PRN $\mathrm{O}_{2}$ ) or severe (140-d/100\% $\mathrm{O}_{2}$ ) BPD. Immunoblotting was performed for $\alpha 1$-AT using a polyclonal antibody. A representative immunoblot is shown. Black and white arrows indicate $52 \mathrm{kD}$ native $\alpha 1$-AT and cleaved $\alpha 1$-AT, respectively. 
Synthesis of al-antitrypsin in baboon lung and liver tissues. There are three major mechanisms that can lead to increased levels of $\alpha 1$-AT, a plasma serpin, in the airways of baboons with BPD. The first is the leakage of the protein into the airways as a result of impaired alveolar-capillary integrity, a well-known feature of several inflammatory lung diseases including BPD. Other possibilities include increased local production of $\alpha 1$-AT, for example, by airway epithelial cells, and increased synthesis in the liver. To explore the latter two possibilities, we performed real-time PCR for $\alpha 1$-AT using baboon lung and liver cDNA samples. The relative steadystate mRNA levels of $\alpha 1$-AT in the lung were very low without any major differences among the groups (data not shown). In the liver, $\alpha 1$-AT mRNA levels were comparable in 140-d GC animals and BPD animals and higher than full-term animals; however, this difference did not reach statistical significance (Fig. $2, p=0.05$ ). In the 140-d severe BPD group, $\alpha 1$-AT mRNA levels were lower than in the 125-d new BPD group, but this difference also did not reach statistical significance $(p=$ 0.05 ). These data suggest that alterations in lung or liver mRNA synthesis of $\alpha 1$-AT are unlikely to account for the increased levels of $\alpha 1$-AT in the airways in BPD.

Functional activity of airway al-antitrypsin in BPD. Proteolytic degradation and oxidative inactivation are two major mechanisms that regulate the activity of serpins. We detected a $52 \mathrm{kD}$ full-length $\alpha 1$-AT molecule in all BPD BALF samples along with some cleaved forms. The $52 \mathrm{kD}$ band could represent either functionally active native $\alpha 1$-AT or its inactive oxidized form. To determine the functional activity of $\alpha 1$-AT, we incubated BALF samples with exogenous NE and assessed whether $\alpha 1$-AT formed SDS-stable high molecular mass complexes with NE (Fig. $3 A$ and Table 1). This method allowed us to specifically assess the NE-neutralizing activity of $\alpha 1$-AT rather than other potential inhibitors of NE in BALF, such as SerpinB1 (30). All full-term control BALF samples demonstrated the presence of functionally active $\alpha 1$-AT by formation of a high molecular mass complex upon

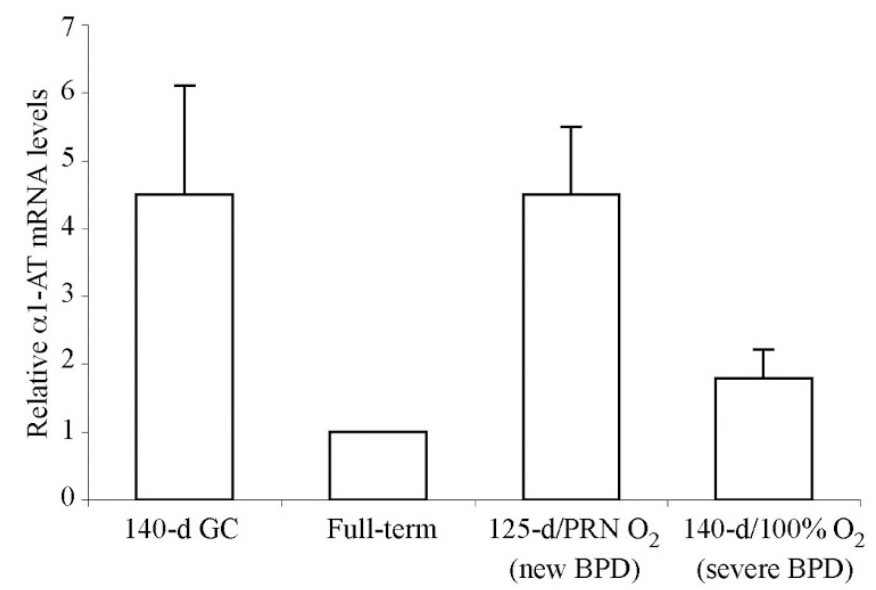

Figure 2. Steady-state relative mRNA levels of $\alpha 1$-AT in baboon liver samples. Relative steady-state mRNA levels of $\alpha 1$-AT in liver tissues were determined by real-time RT-PCR using RNA isolated from 140-d GC, full-term (FT), new (125-d/14 d PRN $\mathrm{O}_{2}$ ) and severe (140-d/100\% $\mathrm{O}_{2}$ ) BPD group baboons. $n=4-5$ animals/group. Data are expressed as mean \pm SEM from two independent experiments performed in triplicates.

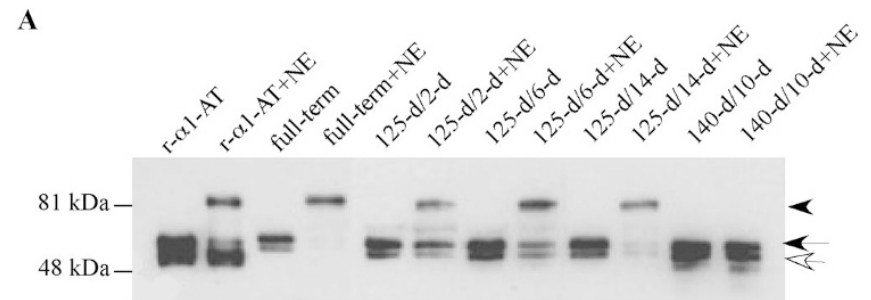

B

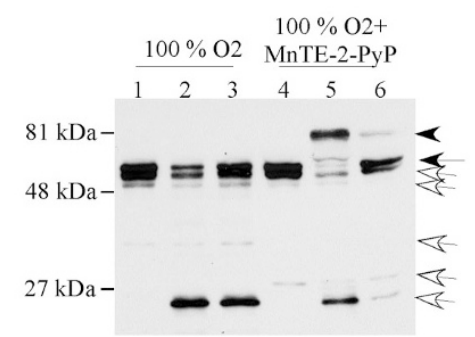

Figure 3. Assessment of antineutrophil elastase activity by formation of high molecular mass complexes between $\alpha 1$-AT and exogenous neutrophil elastase in baboon BALF samples. (A) Recombinant $\alpha 1$-AT or baboon necropsy BALF samples were incubated with PBS or purified NE (32 ng) at RT for $15 \mathrm{~min}$, then exposed to SDS-PAGE and immunoblotting using a polyclonal antibody for $\alpha 1$-AT under reducing conditions. A representative immunoblot is shown. Arrowhead, black arrow, and white arrow indicate 81 $\mathrm{kD}$ complexed $\alpha 1$-AT, $52 \mathrm{kD}$ native $\alpha 1$-AT, and cleaved $\alpha 1$-AT, respectively. (B) Representative immunoblot demonstrating $\alpha 1$-AT immunoreactivity in necropsy BALF samples of a baboon delivered at $140 \mathrm{~d}$ gestation and treated with mechanical ventilation and $100 \% \mathrm{O}_{2}$ for $10 \mathrm{~d}$ and a baboon that received i.v. antioxidant MnTE-2-PyP for $10 \mathrm{~d}$. Samples were incubated at RT for 15 min with PBS (lanes 1 and 4) or two different amounts of purified NE (32 ng, lanes 2 and 5 , or $8 \mathrm{ng}$, lanes 3 and 6 ). Immunoblotting was performed under reducing conditions using a polyclonal antibody against $\alpha 1$-AT.

incubation with NE (Fig. 3A, arrowhead). In the new BPD group, we tested the elastase inhibitory activity of BALF samples obtained after $2 \mathrm{~d}(n=3), 6 \mathrm{~d}(n=3)$, and $14 \mathrm{~d}(n=$ 5) of ventilation and $\mathrm{PRN} \mathrm{O}_{2}$ treatment. All 2-d and 6-d samples and $80 \%$ of the 14-d BALF samples contained functionally active $\alpha 1$-AT as indicated by the detection of high molecular mass bands (Fig. 3A, arrowhead). In contrast, high molecular mass complexes were detected in only $33 \%$ of the BALF samples from hyperoxia-induced severe BPD group animals $(n=6)$. Remarkably, all BALF samples obtained from hyperoxia-exposed and MnTE-2-PyP-treated baboons demonstrated the presence of $\alpha 1$-AT with NE-inhibitory activity as evidenced by formation of high molecular mass complexes upon incubation with $\alpha 1$-AT $(n=7, p<0.05$ versus $100 \% \mathrm{O}_{2}$ treated group, Fisher's exact test, Fig. $3 B$ ).

\section{DISCUSSION}

In this study, we determined the relative antigenic and functional levels of airway $\alpha 1$-AT in two different baboon models of BPD and evaluated the effect of a catalytic antioxidant, MnTE-2-PyP, on the activity of $\alpha 1$-AT in the "severe BPD" model. An imbalance between NE and its major endogenous inhibitor $\alpha 1$-AT was proposed to play a role in the development of BPD more than 25 y ago (31). Since then, major advances in neonatology have led to a significant 
Table 1. Summary of $\alpha 1$-antitrypsin activity in necropsy bronchoalveolar lavage samples of baboons with BPD

\begin{tabular}{|c|c|c|c|c|c|}
\hline \multirow[b]{2}{*}{ BALF sample } & \multicolumn{3}{|c|}{ 125-d gestation/PRN $\mathrm{O}_{2}$ model (new BPD) } & \multicolumn{2}{|c|}{$140 \mathrm{~d} / 100 \% \mathrm{O}_{2}$ model (severe BPD) } \\
\hline & $\begin{array}{c}2-\mathrm{d} \\
(n=3)\end{array}$ & $\begin{array}{c}6-\mathrm{d} \\
(n=3)\end{array}$ & $\begin{array}{c}14-\mathrm{d} \\
(n=5)\end{array}$ & $\begin{array}{c}\text { Untreated } \\
(n=6)\end{array}$ & $\begin{array}{c}\text { MnTE-2-PyP-treated } \\
(n=7)\end{array}$ \\
\hline Activity of $\alpha 1-\mathrm{AT}^{*}(\%)$ & 100 & 100 & 80 & 33 & $100 \dagger$ \\
\hline
\end{tabular}

* Assessed by formation of high molecular mass complexes of $\alpha 1$-AT when treated with neutrophil elastase.

$\dagger p<0.05 v s$ untreated group (Fisher's exact test).

improvement in the clinical severity but not in the incidence of this disease. In the majority of surfactant-treated baboon and human infants with evolving BPD, NE is no longer the major offending protease $(16,30,32,33)$. Consistent with these observations, we found increased levels of functionally active $\alpha 1$-AT in the airways in the new BPD model, which indicates the presence of adequate elastase inhibitory activity. Our results also suggest that the increased airway levels of $\alpha 1$-AT in BPD are likely due to increased alveolar-capillary permeability rather than increased transcription of $\alpha 1$-AT in the lung or liver in this model although we cannot rule out the possibility of increased mRNA or protein stability.

In baboon BALF samples, we detected only native and cleaved, but not complexed forms of $\alpha 1$-AT. The presence of cleaved forms of $\alpha 1$-AT can be explained by three potential mechanisms: cleavage of $\alpha 1$-AT by noncognate proteases, such as cysteine proteases; cleavage of oxidized $\alpha 1$-AT by target proteases, such as $\mathrm{NE}$; and cleavage of the $4 \mathrm{kD}$ $\mathrm{COOH}$-terminal fragment as a result of complex formation between $\alpha 1$-AT and a target protease, thus yielding a $\sim 48 \mathrm{kD}$ cleaved protein. In untreated BALF samples, some of the cleaved forms of $\alpha 1$-AT were the same size as the cleaved $\alpha 1$-AT (48 kD) after in vitro complex formation between recombinant $\alpha 1$-AT and purified NE (Fig. 3). This suggests that these samples might have contained complexed $\alpha 1$-AT that was subsequently degraded during the retrieval process or storage of BALF.

Despite all advances in neonatology, a subgroup of extremely LBW infants continues to develop severe BPD after RDS that may be complicated by sepsis, medical treatmentrefractory patent ductus arteriosus, and/or pulmonary interstitial emphysema (34). The severe inflammatory response and treatment with high concentrations of oxygen in these fragile patients are inevitably associated with production of abundant reactive oxygen species and oxidant injury $(15,35)$. Our findings in the "severe BPD" model indicate that although the airway antigenic levels of $\alpha 1$-AT are similar to those in the new BPD model, the $\alpha 1$-AT recovered from the BALF of these animals does not have adequate elastase inhibitory activity as it fails to form SDS-stable complexes with exogenous NE. Because the majority of airway $\alpha 1$-AT in severe BPD was detected as a full-length $52 \mathrm{kD}$ band by immunoblotting, we reasoned that oxidative inactivation was a likely cause for failure of $\alpha 1$-AT to form inhibitory complexes with NE in these samples. Oxidation of methionine in P1 position of $\alpha 1$-AT converts this amino acid to Met-SO, thus resulting in a dramatic loss of functional activity (36). In a study that examined BAL protein oxidation in children with chronic lung disease, $\alpha 1-\mathrm{AT}$ was identified as one of the most sensitive proteins to oxidation in the airways (37). In another study, inactivation of $\alpha 1$-AT by smokers' macrophages was prevented by addition of antioxidant enzymes such as superoxide dismutase and catalase (38). Consistent with our hypothesis and these previous studies, we detected active $\alpha 1-\mathrm{AT}$ in BALF of a significantly higher number of severe BPD group baboons treated with the antioxidant MnTE-2-PyP compared with those who did not receive this treatment.

In summary, antioxidant treatment of baboons with severe BPD resulted in augmentation of the elastase inhibitory activity of airway $\alpha 1$-AT. Protection of the RSL of $\alpha 1$-AT against oxidative inactivation may be beneficial for antiproteinase and antiapoptotic effects of this serpin, both of which are relevant for the pathogenesis of BPD. Further studies are needed to determine whether preservation of the function of $\alpha 1$-AT is one of the potential mechanisms that contribute to the therapeutic effects of antioxidants in animal models of BPD and whether this approach could benefit human infants with evolving severe BPD.

Acknowledgments. We thank Dr. Richard Parad for his critical review of the manuscript. We also thank Vickie Winter and Dr. Jackie Coalson for provision of the baboon samples.

\section{REFERENCES}

1. Van Marter LJ 2009 Epidemiology of bronchopulmonary dysplasia. Semin Fetal Neonatal Med 14:358-366

2. Baraldi E, Filippone M 2007 Chronic lung disease after premature birth. N Engl J Med 357:1946-1955

3. Bland RD 2005 Neonatal chronic lung disease in the post-surfactant era. Biol Neonate 88:181-191

4. Eichenwald EC, Stark AR 2008 Management and outcomes of very low birth weight. N Engl J Med 358:1700-1711

5. Jobe AH 2011 The new bronchopulmonary dysplasia. Curr Opin Pediatr 23:167-172

6. Doyle LW 2006 Respiratory function at age 8-9 years in extremely low birthweight/ very preterm children born in Victoria in 1991-1992. Pediatr Pulmonol 41:570-576

7. Chye JK, Gray PH 1995 Rehospitalization and growth of infants with bronchopulmonary dysplasia: a matched control study. J Paediatr Child Health 31:105-111

8. Greenough A 2007 Late respiratory outcomes after preterm birth. Early Hum Dev 83:785-788

9. Kinsella JP, Greenough A, Abman SH 2006 Bronchopulmonary dysplasia. Lancet 367:1421-1431

10. Jeng SF, Hsu CH, Tsao PN, Chou HC, Lee WT, Kao HA, Hung HY, Chang JH, Chiu NC, Hsieh WS 2008 Bronchopulmonary dysplasia predicts adverse developmental and clinical outcomes in very-low-birthweight infants. Dev Med Child Neurol 50:51-57

11. Anderson PJ, Doyle LW 2006 Neurodevelopmental outcome of bronchopulmonary dysplasia. Semin Perinatol 30:227-232

12. Speer CP 2009 Chorioamnionitis, postnatal factors and proinflammatory response in the pathogenetic sequence of bronchopulmonary dysplasia. Neonatology 95:353361

13. Arnon S, Grigg J, Silverman M 1993 Pulmonary inflammatory cells in ventilated preterm infants: effect of surfactant treatment. Arch Dis Child 69:44-48

14. Groneck P, Gotze-Speer B, Oppermann M, Eiffert H, Speer CP 1994 Association of pulmonary inflammation and increased microvascular permeability during the development of bronchopulmonary dysplasia: a sequential analysis of inflammatory mediators in respiratory fluids of high-risk preterm neonates. Pediatrics 93:712-718

15. Bose CL, Dammann CE, Laughon MM 2008 Bronchopulmonary dysplasia and inflammatory biomarkers in the premature neonate. Arch Dis Child Fetal Neonatal Ed 93:F455-F461 
16. Altiok O, Yasumatsu R, Bingol-Karakoc G, Riese RJ, Stahlman MT, Dwyer W, Pierce RA, Bromme D, Weber E, Cataltepe S 2006 Imbalance between cysteine proteases and inhibitors in a baboon model of bronchopulmonary dysplasia. Am J Respir Crit Care Med 173:318-326

17. Didrik Saugstad O 2010 Oxygen and oxidative stress in bronchopulmonary dysplasia. J Perinat Med 38:571-577

18. Ahmed MN, Suliman HB, Folz RJ, Nozik-Grayck E, Golson ML, Mason SN, Auten RL 2003 Extracellular superoxide dismutase protects lung development in hyperoxia-exposed newborn mice. Am J Respir Crit Care Med 167:400-405

19. Auten RL, O'Reilly MA, Oury TD, Nozik-Grayck E, Whorton MH 2006 Transgenic extracellular superoxide dismutase protects postnatal alveolar epithelial proliferation and development during hyperoxia. Am J Physiol Lung Cell Mol Physiol 290:L32L40

20. Chang LY, Subramaniam M, Yoder BA, Day BJ, Ellison MC, Sunday ME, Crapo JD 2003 A catalytic antioxidant attenuates alveolar structural remodeling in bronchopulmonary dysplasia. Am J Respir Crit Care Med 167:57-64

21. Davis JM, Parad RB, Michele T, Allred E, Price A, Rosenfeld W 2003 Pulmonary outcome at 1 year corrected age in premature infants treated at birth with recombinant human $\mathrm{CuZn}$ superoxide dismutase. Pediatrics 111:469-476

22. Stiskal JA, Dunn MS, Shennan AT, O'Brien KK, Kelly EN, Koppel RI, Cox DW, Ito S, Chappel SL, Rabinovitch M 1998 alpha1-Proteinase inhibitor therapy for the prevention of chronic lung disease of prematurity: a randomized, controlled trial. Pediatrics 101:89-94

23. Zhang B, Lu Y, Campbell-Thompson M, Spencer T, Wasserfall C, Atkinson M, Song S 2007 Alpha1-antitrypsin protects beta-cells from apoptosis. Diabetes 56:1316-1323

24. Petrache I, Fijalkowska I, Medler TR, Skirball J, Cruz P, Zhen L, Petrache HI, Flotte TR, Tuder RM 2006 alpha-1 antitrypsin inhibits caspase-3 activity, preventing lung endothelial cell apoptosis. Am J Pathol 169:1155-1166

25. Petrache I, Fijalkowska I, Zhen L, Medler TR, Brown E, Cruz P, Choe KH, Taraseviciene-Stewart L, Scerbavicius R, Shapiro L, Zhang B, Song S, Hicklin D, Voelkel NF, Flotte T, Tuder RM 2006 A novel antiapoptotic role for alpha1antitrypsin in the prevention of pulmonary emphysema. Am J Respir Crit Care Med 173:1222-1228

26. Coalson JJ, Winter VT, Siler-Khodr T, Yoder BA 1999 Neonatal chronic lung disease in extremely immature baboons. Am J Respir Crit Care Med 160:1333-1346
27. Coalson JJ, Winter VT, Gerstmann DR, Idell S, King RJ, Delemos RA 1992 Pathophysiologic, morphometric, and biochemical studies of the premature baboon with bronchopulmonary dysplasia. Am Rev Respir Dis 145:872-881

28. VanGuilder HD, Vrana KE, Freeman WM 2008 Twenty-five years of quantitative PCR for gene expression analysis. Biotechniques 44:619-626

29. Ghelfi E, Karaaslan C, Berkelhamer S, Akar S, Kozakewich H, Cataltepe S 2010 Fatty acid binding proteins and peribronchial angiogenesis in bronchopulmonary dysplasia. Am J Respir Cell Mol Biol doi:10.1165/rcmb.2010-0376OC

30. Yasumatsu R, Altiok O, Benarafa C, Yasumatsu C, Bingol-Karakoc G, RemoldO'Donnell E, Cataltepe S 2006 SERPINB1 upregulation is associated with in vivo complex formation with neutrophil elastase and cathepsin $\mathrm{G}$ in a baboon model of bronchopulmonary dysplasia. Am J Physiol Lung Cell Mol Physiol 291:L619-L627

31. Merritt TA, Cochrane CG, Holcomb K, Bohl B, Hallman M, Strayer D, Edwards DD, Gluck L 1983 Elastase and alpha 1-proteinase inhibitor activity in tracheal aspirates during respiratory distress syndrome. Role of inflammation in the pathogenesis of bronchopulmonary dysplasia. J Clin Invest 72:656-666

32. Sluis KB, Darlow BA, Vissers MC, Winterbourn CC 1994 Proteinase-antiproteinase balance in tracheal aspirates from neonates. Eur Respir J 7:251-259

33. Cederqvist K, Siren V, Petaja J, Vaheri A, Haglund C, Andersson S 2006 High concentrations of plasminogen activator inhibitor-1 in lungs of preterm infants with respiratory distress syndrome. Pediatrics 117:1226-1234

34. Smith VC, Zupancic JA, McCormick MC, Croen LA, Greene J, Escobar GJ, Richardson DK 2005 Trends in severe bronchopulmonary dysplasia rates between 1994 and 2002. J Pediatr 146:469-473

35. Schock BC, Sweet DG, Halliday HL, Young IS, Ennis M 2001 Oxidative stress in lavage fluid of preterm infants at risk of chronic lung disease. Am J Physiol Lung Cell Mol Physiol 281:L1386-L1391

36. Potempa J, Korzus E, Travis J 1994 The serpin superfamily of proteinase inhibitors: structure, function, and regulation. J Biol Chem 269:15957-15960

37. Starosta V, Griese M 2006 Protein oxidation by chronic pulmonary diseases in children. Pediatr Pulmonol 41:67-73

38. Hubbard RC, Ogushi F, Fells GA, Cantin AM, Jallat S, Courtney M, Crystal RG 1987 Oxidants spontaneously released by alveolar macrophages of cigarette smokers can inactivate the active site of alpha 1-antitrypsin, rendering it ineffective as an inhibitor of neutrophil elastase. J Clin Invest 80:1289-1295 